\title{
Epidermolysis Bullosa
}

National Cancer Institute

\section{Source}

National Cancer Institute. Epidermolysis Bullosa. NCI Thesaurus. Code C67383.

An autosomal recessive inherited skin disorder caused by mutations in the genes

encoding keratins 5 and 14 , collagen VII or laminin 5 . It is characterized by skin fragility and the formation of blisters. The blisters may become large and ulcerated, resulting in skin infections and loss of body fluids. 